\title{
Globe
}

Revue internationale d'études québécoises

\section{Louise Beaudoin et Stéphane Paquin (dir.) : Pourquoi la Francophonie?, Montréal, VLB éditeur, 2008}

\section{Tristan Leperlier}

Volume 12, numéro 2, 2009

URI : https://id.erudit.org/iderudit/1000719ar

DOI : https://doi.org/10.7202/1000719ar

Aller au sommaire du numéro

Éditeur(s)

Globe, Revue internationale d'études québécoises

ISSN

1481-5869 (imprimé)

1923-8231 (numérique)

Découvrir la revue

Citer ce compte rendu

Leperlier, T. (2009). Compte rendu de [Louise Beaudoin et Stéphane Paquin (dir.) : Pourquoi la Francophonie?, Montréal, VLB éditeur, 2008]. Globe, 12(2), 216-225. https://doi.org/10.7202/1000719ar d'utilisation que vous pouvez consulter en ligne.

https://apropos.erudit.org/fr/usagers/politique-dutilisation/ 


\section{Louise Beaudoin et Stéphane Paquin (dir.) \\ Pourquoi la Francophonie? \\ Montréal, VLB éditeur, 2008.}

Ce collectif présente un certain nombre de perspectives sur la crise de légitimité que traverse actuellement la Francophonie. Les auteurs s'entendent sur le caractère positif des fondements idéologiques de la Francophonie, et également sur les causes de la crise que traverse l'institution: un défaut de communication qui fait de la Francophonie la proie des préjugés; une attitude négative de la France; ou encore des réalisations encore trop circonscrites ou peu publicisées. Les collaborateurs de cet ouvrage proposent ainsi à l'institution francophone de nombreux projets et objectifs nouveaux. Une grande majorité des collaborateurs réclame ainsi un recentrement de l'institution sur sa dimension originelle de coopération culturelle et technique, plutôt que le renforcement de la part de la diplomatie et de la politique. Quelques auteurs (Michel Guillou, Jean Tabi Manga, Christian Philip) demandent enfin que la Francophonie mène une véritable réflexion sur une politique de coopération économique, seule à même de soutenir les efforts entrepris dans les autres domaines. La faiblesse relative des propositions de nos auteurs à ce sujet et l'absence presque totale de cette thématique lors du Sommet de Québec montrent bien que c'est dans ce domaine de coopération que le plus dur reste à faire.

\section{DES ARTICLES DE SPÉCIALISTES POUR SPECIALISTES}

Ce collectif publié à la fin de l'année 2008 à Montréal possède a priori tout pour attirer le lecteur intéressé par la Francophonie: de grands noms familiers au lecteur (Abdou Diouf, Louise Beaudoin, Alain Juppé), de grands spécialistes (Michel Guillou, Françoise Massart-Piérard), d'origines géographiques diverses (France, Québec, Cameroun, Liban) et de disciplines tout aussi diverses (politique, sociologie, droit, anthropologie). On pourra regretter toutefois un manque de synthèse dans cet ouvrage, surtout quand il s'agit d'éclairer le titre Pourquoi la Francophonie?, rôle dévolu aux quelques courtes lignes de la quatrième de couverture. L'impression générale que l'on retire est celle d'une simple juxtaposition d'articles ${ }^{3}$. C'est le Secrétaire Général de

$$
+4
$$

3. Au point que certains articles peuvent paraître à l'ecart du sujet, comme l'analyse socioanthropologique, par ailleurs très intéressante, de Jean-François Payette et de Gérard Boulet: "La Francophonie, plus qu'une question de langue: une comparaison culturelle Québec-France». 
l'Organisation Internationale de la Francophonie (OIF), Abdou Diouf, sorte de primus inter pares dans cette table ronde d'éminents auteurs, qui signe la préface, moins pour synthétiser l'ouvrage que pour exposer la position offcielle de la Francophonie. On sent ici que le public visé est celui des connaisseurs de la question, des convaincus de la Francophonie aux spécialistes, comme le confirme par ailleurs le peu de mentions précises des réalisations concrètes de la Francophonie; cela peut sembler paradoxal pour un ouvrage dont bien des articles mettent en avant la nécessité de rendre accessible la question francophone à un public plus large que celui des seuls politiques et spécialistes.

\section{POURQUOI LA FRANCOPHONIE? : QUEL DÉBAT LORS DE LA PUBLICATION?}

Comme l'indique, de manière maintenant traditionnelle, la majuscule de "Francophonie", l'accent est ici mis sur la dimension institutionnelle de la francophonie, à savoir les institutions transnationales qui cherchent à donner des objectifs communs à des pays qui, pour l'essentiel, font partie de l'OIF. La question posée ici, "Pourquoi la Francophonie? ", n'est pas innocente: il s'agit moins d'exposer les causes, voire les motivations ou les buts de la Francophonie, que de répondre à une polémique: quelle est la légitimité de la Francophonie, actuellement et absolument?

Alors que la Francophonie est née d'une volonté des gouvernements de pays anciennement colonisés (Senghor du Sénégal, Bourguiba de Tunisie, Diori du Niger, notamment), ceux-ci semblent être revenus de ce "rêve" (Michel Guillou). Soit qu'elle apparaisse comme un avatar de la Françafrique (comme pour l'Algérie, qui refuse encore l'adhésion à l'OIF); soit qu'elle semble manquer aux missions qu'elle s'était fixées. En France, la question francophone est considérée comme "ringarde", au point, comme le rappelle la quatrième de couverture, d'avoir été "négligée" lors des élections présidentielles de 2007; ou au mieux, par exemple lors de la récente crise de TV5 de 2007-2008, la francophonie est perçue comme un simple prolongement de la France, donnant par là même du grain à moudre aux détracteurs d'une Francophonie postcoloniale, voire néocoloniale. Le contexte immédiat de la publication de ce livre est toutefois positif, puisque quelques mois avant celle-ci, le 18 mars 2007, était mise en application à l'UNESCO l'importante "Convention sur la protection et la promotion de la diversité des expressions culturelles", pour laquelle la Francophonie avait joué un rôle considérable. Le Sommet des chefs d'États et de gouvernements de la Francophonie à Québec quelques mois plus tard (octobre 2008) aurait pu 
par ailleurs être l'occasion d'entamer les réformes pour sortir de la crise identifiée.

\section{FONDEMENTS IDÉOLOGIQUES DE LA FRANCOPHONIE}

Tous les auteurs s'accordent sur le but, le "pour quoi", et donc les fondements idéologiques de la Francophonie, inscrits dans sa raison d'être depuis sa fondation. Pour Michel Guillou, «l'universalisme francophone se présente comme un tissage de l'idéal républicain français et de la civilisation de l'universel de Senghor. Il est Sud tout autant que Nord " (p. 91). Pour lui la langue française cristallise, au moins fantasmatiquement, les idéaux des Lumières et de la Révolution, permettant à la francophonie un élargissement au-delà de l'histoire coloniale. L'égalité entre les pays étant ainsi inscrite dans l'histoire même de la Francophonie, Abdou Diouf peut écrire : "La Francophonie, aujourd'hui, c'est donc, non seulement, une certaine vision des relations internationales fondée sur le multilatéralisme, mais encore une certaine vision de la coopération fondée sur le dialogue et le respect de la diversité" (p. 16).

\section{EFFICACITE DE LA FRANCOPHONIE?}

Cette "belle idée" n'empêche toutefois pas Katia Haddad de faire une critique extrêmement virulente de l'évolution de l'institution, qu'elle qualifie de "gâchis" (p. 183). Elle estime que la crise actuelle ne résulte globalement que d'un manque de "vision stratégique", qu'elle explique par la crispation des élites francophones sur la lutte contre l'anglais et les États-Unis. Affirmation qui n'apparaît pas complètement infondée à la lecture de certains articles ici rassemblés.

En dehors de ce texte, les actions positives de la Francophonie sont abondamment rappelées. La "Convention sur la protection et la promotion de la diversité des expressions culturelles" signée à l'UNESCO est saluée par la quasi-totalité des collaborateurs. Par son importante contribution à son élaboration, la Francophonie montre qu'elle considère que la culture ne peut être soumise à la loi du marché global, mais qu'elle doit être protégée et promue pour ce qu'elle est, à savoir l'expression de l'homme dans

$$
4+4
$$

4. Que cette philosophie du très francisé Senghor soit proprement africaine n'est pas ici remis en question. 5. On n'a qu'à comparer cet article à celui de l'altermondialiste Bernard Cassen, qui commence son article au titre évocateur, "L'ocanisarion linguistique", avec une attaque en règle contre la domination de l'anglais. 
sa diversitét. Mais en dehors de celle-ci ou de TV5, qui reçoit son tribut d'éloge comme chaîne de télévision internationale francophone multilatérale figurant en deuxième position après $\mathrm{CNN}$, les autres réalisations concrètes de la Francophonie ne sont malheureusement que peu mentionnées dans ces articles, à l'exception de celui proposé par Louise Beaudoin, à la toute fin de l'ouvrage.

De manière très originale, un certain nombre d'articles attire l'attention sur une qualité propre à la Francophonie: l'importance en son sein de la société civile. Jacques Frémont rappelle ainsi que la société civile, formée des représentants de la Fédération internationale des droits de l'Homme, de Reporters sans frontières, des universitaires, etc., a contribué à l'élaboration des textes des Déclarations de Bamako ou de Saint Boniface. Comme le montre Bruno Maltais, l'importance de la vie associative depuis la première moitié du XX' siècle (l'Union internationale des journalistes et de la presse de langue française a été fondée en 1926) est une autre preuve de la vitalité francophone en dehors de sa version institutionnelle et possiblement bureaucratique.

\section{CRISE DE (RE)CONNAISSANCE}

Ce caractère éminemment positif de la Francophonie illustré ici peut rendre paradoxale la crise populaire que tous pointent par ailleurs. La plupart des rédacteurs accordent au préfacier Abdou Diouf que la perception de la Francophonie est tributaire de préjugés en partie illégitimes.

Mais le problème le plus grave réside selon eux dans la méconnaissance de l'action, voire de l'existence même de la F/francophonie. Michel Guillou déclare à propos de ses étudiants de la chaire Senghor que "leur degré d'ignorance du fait francophone est abyssal" (p. 85).

La première réforme à envisager est donc celle de la communication. Guillou, de manière provocatrice, déclare qu'il faut " "vendre" la Francophonie", car le plus important pour lui est de créer un "sentiment d'appartenance ": "construire une communauté de sentiment et d'intérêt" (p. 92-93). À l'instar de Christian Philip, il énumère un certain nombre de mesures pratiques à adopter, comme la création d'une forme d'"ERASMUS francophone".

$$
+4
$$

6. Ce qui n'est pas incompatible avec des stratégies économiques de défense d'industries culturelles nationales, en particulier celles de la France et du Canada. 
Cette méconnaissance ne saurait toutefois tout expliquer, et c'est pourquoi l'ensemble des collaborateurs de ce collectif tente de repérer avec précision les difficultés structurelles de la Francophonie, et d'y apporter des solutions.

\section{LA PLACE DE LA FRANCE}

On retire de la lecture de ce livre un intéressant paradoxe sur la position de la France par rapport à la francophonie. D'une part, nombreux sont les auteurs, comme Christian Philip, à critiquer la tendance des élites françaises à abandonner la défense du français comme combat politique, mais aussi dans leur comportement à l'etranger, voire en France.

Mais, d'autre part, l'attitude de la France est également remise en cause quand son intérêt pour la francophonie se fait trop marqué. En effet, la politique du plus grand pays francophone ne peut que rejaillir sur la réputation de la Francophonie. Certains dénoncent à mots couverts la politique de la «Françafrique». D’autres, comme Alain Juppé, en appellent à une attitude plus humble vis-à-vis de ses autres partenaires francophones. Catherine Tasca revient sur la crise de TV5 ${ }^{7}$, qui montre bien selon elle la « conception uniquement utilitariste de la francophonie " (p. 82) véhiculée par la France. Mais le taux de participation de celle-ci au financement des différents organismes de la Francophonie ( $84 \%$ des dépenses de TV5 d'après Louise Beaudoin [p. 210]) lui donne un droit de regard bien plus important que l'idéologie de la stricte égalité entre les membres de l'OIF ne l'autorise. C'est pourquoi Christian Philip demande en conclusion de son article que les "autres pays membres, du Nord et du Sud, prennent une place plus importante dans le financement et les responsabilités de nos institutions communes" (p. 171).

\section{QUELLE IDENTITÉ POUR LA FRANCOPHONIE?}

Si les principes fondamentaux sont acquis pour l'ensemble des promoteurs de la Francophonie, ce collectif montre que la ligne directrice de l'institution francophone peut être l'objet d'importants débats. Le domaine que, dans le cadre de ces fondements, la Francophonie doit privilégier, varie selon les auteurs: culturel et technique, politique, ou encore économique. Stéphane

$$
++*
$$

7. Elle a eu pour origine le projet du gouvernement français d'intégrer la chaîne multilatérale francophone à un ensemble, "France Monde", qui aurait regroupé entre autres France 24 et RFI, qui sont, elles, exclusivement françaises. Face au tollé général des autres partenaires, le gouvernement français a dû reculer. 
Paquin, comme une majorité de nos spécialistes, met en question l'évolution de la Francophonie vers trop de politique: «[...] d'essentiellement technique l'OIF devient de plus en plus politique ${ }^{8}[\ldots]$. [E]st-ce que la Francophonie pourra réussir là où d'autres organisations internationales n'ont pas connu de véritables succès?" (p. 30).

On voit que c'est l'identité même de la Francophonie qui est ici en jeu : qu'est-ce qui doit la distinguer d'autres organisations internationales? Jacques Frémont, à l'inverse, est partisan de la ligne politique que semble actuellement privilégier l'institution:

Les sujets relatifs à la langue, à la pluralité culturelle, à l'enseignement ou encore au développement durable, pour ne prendre que ces exemples, s'ils sont importants, à terme ne sauront justifier à eux seuls l'existence d'une Francophonie institutionnelle regroupant plus d'une cinquantaine d'États et gouvernements. [...] En bref, la Francophonie sera politique et elle s'attachera à la protection et au développement de la démocratie et des droits et libertés et à la paix, ou elle ne sera tout simplement pas (p. 132).

Il montre ainsi que l'identité de la Francophonie est étroitement liée à ses membres, dont le nombre s'est considérablement élargi depuis la création de l'institution'. On comprend dès lors l'importance de la question du statut de chacun d'eux au sein de l'OIF, et celle de l'adhésion de nouveaux membres (en dehors des deux pays réputés francophones que sont l'Algérie et Israël) afin d'éviter l'effet de "ticket gratuit» dénoncé par plusieurs.

\section{LA PROMOTION DE LA LANGUE FRANÇAISE ET DE LA DIVERSITÉ CULTURELLE ET LINGUISTIQUE}

Dans la mesure où ils sont en majorité partisans d'une ligne (et donc d'une identité) plus culturelle de la Francophonie, les auteurs de cet ouvrage formulent l'essentiel de leurs propositions autour de cette question.

"Le volet défensif" réside pour Bernard Cassen dans l'application à tous les niveaux de dispositifs de protection de la langue comme, au niveau

$$
+\div
$$

8. Avant de s'appeler Organisation internationale de la Francophonie, la Francophonie inscitutionnelle était l'Agence de coopéracion culturelle et technique, I'ACCT. Le Sommer de Cotonou de 1995 a décidé que le moment érait venu de donner à la Francophonie sa pleine dimension politique.

9. De 43 membres en 1986, elle est passée à 68 (dont 13 observateurs) aujourd'hui, donc bien au-delà du cercle colonial de la France et de la Belgique. C'est l'adhésion de nouveaux membres pour lesquels le français est d'importance mineure (l'Albanie, la Macédoine, la Moldavie... voire la Pologne et l'Ukraine pour les observateurs) qui pose probleme pour nos auteurs. 
national, la loi Toubon en France ou la loi 101 au Québec. Jean-François Lisée défend quant à lui la création d'un "traité international sur la diversité linguistique" qui irait plus loin que la Convention sur la diversité culturelle, car " la logique marchande prime sur (sic.) la logique identitaire ou linguistique dans les grandes enceintes dont [le Québec et le Canada sont membres]" (p. 117 et 115).

L'enseignement du français et en français (où Internet joue un rôle central, entre autres par le biais de "l'université numérique francophone mondiale») apparaît comme nécessaire, mais non suffisant, en particulier dans une philosophie globale de la diversité culturelle; ce qui explique la promotion du multilinguisme par nos auteurs. Pour Alain Juppé, l'enseignement réel de deux langues étrangères dans l'Union européenne serait en outre positif pour le français. Michel Guillou revient sur la création de classes bilingues qui avaient suscité, selon lui, beaucoup d'espoir au Vietnam avant que le projet ne tombe à l'eau, et avance même l'idée de classes trilingues (avec l'anglais). Il estime en effet que la promotion du français là où il est en difficulté ne pourra se faire que si l'on parvient à convaincre qu'elle est une langue «à débouchés". Il faut pour cela favoriser les réseaux d'élites, renforcer les relations avec la société civile francophone et les échanges universitaires.

Un certain nombre de spécialistes ici rassemblés se prononce pour une voie assez originale: pour Bernard Cassen, le "volet offensif» du combat pour le français passe par le développement de l'enseignement de l'intercompréhension entre les langues romanes ${ }^{10}$. Aurélien Yannick en fait même l'objet de son article: la maîtrise d'une langue romane deviendrait alors la passerelle d'accès à plusieurs autres, et renforcerait leur attractivité.

Au-delà de l'enseignement de langues, la présence médiatique du français paraît incontournable. Pierre Lampron prône une réforme de TV5, qui a dû son succès à sa facture innovante dans les années 1980 , mais doit aujourd'hui s'adapter à la demande d'interactivité pour le conserver. Jean Tabi Manga et Michel Guillou réclament la création d'un organisme de soutien à la création culturelle francophone, sur le modèle de la Société pour le développement des entreprises culturelles québécoise (SODEC).

$$
+4
$$

10. Des méthodes sont actuellement testées pour enseigner très rapidement et simultanément d'autres langues cypologiquement proches, ce qui est le cas des langues romanes. Les locuteurs parleraient dans leur propre langue, et seraient compris par un autre "romanophone" qui en ferait autant. 


\section{LA POLITIQUE: DEMOCRATIE, PAIX, ÉTAT DE DROIT}

Le très intéressant article du juriste Jacques Frémont fait comprendre en quoi la Déclaration de Bamako de 2000 sur la démocratie et la paix, puis celle de Saint Boniface de 2006 sur la prévention des conflits et la sécurité humaine apportent des éléments véritablement nouveaux par rapport aux textes internationaux antérieurs. Contre l'idée courante selon laquelle cet outil normatif ne ferait que redoubler ceux de l'ONU, Frémont suggère que, outre le fait que ces Déclarations donnent à la Francophonie des moyens idéologiques d'action politique internationale, elles permettent également symboliquement de lier des valeurs à une langue et à son partage, ce qui apparaît d'autant plus justifié pour le français, qui conserve dans les esprits, comme le rappelle Michel Guillou, une force d'attrait liée à l'idéal révolutionnaire et républicain. Par un coup de force de la pensée, partager le français revient donc aussi à partager des valeurs; ou du moins doit y conduire...

Par ailleurs, contre les détracteurs de cette ligne "politiste» qui ne saurait être vraiment efficace du fait des moyens limités de la Francophonie, il estime que, parmi les principes qui devraient guider ses choix, on retrouve celui de la plus-value que son action doit apporter. Il est en effet inutile de dupliquer ce que d'autres font et souvent mieux. Il serait par contre particulièrement profitable que la Francophonie puisse compléter les actions d'autres acteurs internationaux et, dans certains cas, puisse même les coordonner, apportant ainsi une contribution inédite et réellement efficace. Les interventions des dernières années en Haïti, en Mauritanie, en République démocratique du Congo ou au Togo, pour ne mentionner que celles-là, sont des illustrations de cette valeur ajoutée qui représente une condition sine qua non de la pertinence de la Francophonie.

\section{À QUAND UNE COOPÉRATION ÉCONOMIQUE?}

Malgré des sommets comme celui de Québec en 1987 ou d'Hanoi en 1997, la Francophonie ne semble pas être parvenue à mettre en place une coopération économique à l'échelle francophone. Cette incompétence dans un secteur aussi central et visible est un des facteurs autorisant l'opinion publique francophone à parler d'inefficacité à son propos. Cela serait dû à des difficultés structurelles, comme le rappelle Bruno Maltais: «La dispersion géographique des pays francophones, leur disparité de niveau de développement, leur différence en matière de système économique et leur appartenance à d'autres regroupements économiques et commerciaux sont des obstacles à la construction d'un espace économique francophone" (p. 39); mais aussi au fait que les pays du Nord francophone préferent 
réserver ce qui reste de leur budget d'aide au développement aux cadres bilatéraux, plus profitables dans les jeux d'influence politique.

Or, comme l'écrit Jean Tabi Manga, "sans le développement économique, les politiques de diversité culturelle, les avancées démocratiques dans la construction d'un État de droit restent fragiles, aléatoires» (p. 179). Les propositions économiques de nos spécialistes ne sont toutefois pas à la hauteur des attentes, preuve que cette dimension n'est qu'en germe au sein de la Francophonie. Christian Philip, conseiller personnel de Nicolas Sarkozy sur la Francophonie, n'insiste que sur le développement des relations entre les entreprises francophones, par exemple au sein du Forum francophone des affaires. On reste sceptique quant à l'efficacité de cette coopération lorsqu'on connaît l'importante proportion de Pays les moins avancés (PMA) au sein de la Francophonie et la difficulté de ceux-ci à avoir des entreprises propres à "coopérer" dans quelque cadre que ce soit. Michel Guillou mentionne l'éventuelle création de l'Institut francophone de l'économie, qui aurait pour mission de rassembler les actions éparses actuelles de l'OIF, pour mettre en route, dans ses différents volets, la coopération économique dans la Francophonie. Il agirait tout particulièrement dans les secteurs au cœur de la Francophonie: l'éducation, la culture, le développement durable et solidaire. Il disposerait d'un fond d'investissement et de garantie.

$\mathrm{Ne}$ faudrait-il pas réfléchir aussi, parallèlement à ce que proposent ces deux auteurs, à la promotion à grande échelle des initiatives susceptibles de développer l'économie locale, comme les banques de micro-crédit? On s'étonne que la publicité d'un tel système n'ait pas touché les spécialistes et responsables de la Francophonie.

Si l'intérêt des points de vue présentés est indéniable, on pourra regretter dans l'ensemble un manque de concertation dans le choix des collaborateurs. Ceux-ci ne se font que partiellement le reflet des débats au sein de la francophonie, institutionnelle en particulier. En effet, en dehors de Jacques Frémont et d'Abdou Diouf, la défense de la ligne politique n'est que peu représentée; et si plusieurs auteurs revendiquent certes un renforcement de la ligne économique, ils sont trop peu spécialisés en la matière pour formuler des propositions vraiment innovantes. Reste à savoir si les propositions des collaborateurs de Pourquoi la Francophonie? ont trouvé un écho suffisant lors de la "XII" Conférence des chefs d'État et de gouvernement des pays ayant le français en partage ", soit le Sommet de Québec tenu du 17 au 19 octobre 2008. Contrairement au Sommet précédent (à Bucarest), consacré plus spécifiquement aux technologies de l'information dans l'éducation, celui-ci n'est pas parvenu à trouver une ligne directrice qui 
satisfasse tous les membres. La part réservée à la politique reste extrêmement importante, tant dans la Déclaration que dans les Résolutions. Il est à noter par ailleurs une insistance très nette sur la défense et la promotion du français; le secrétaire général est par exemple invité à " entamer les démarches visant à conclure un pacte linguistique avec les États ou gouvernements qui le souhaitent" (Résolution sur la langue française, adoptée lors du Sommet de Québec, http://www.francophonie.org/IMG/pdf/resolution-quebec-20083.pdf [26 janvier 2010]), ce qui peut apparaître comme une première étape, non suffisante certes mais nécessaire, vers un renforcement de l'identité culturelle de la Francophonie. Entièrement oubliée des rédacteurs de ce collectif, la question de l'environnement est très présente dans la Déclaration. En revanche, la dimension économique reste clairement le parent pauvre des projets de la Francophonie, surtout étant donné les enjeux soulevés dans ce livre: il est par exemple indiqué que l'assemblée prendra, "lors du $\mathrm{XIII}^{\mathrm{e}}$ Sommet, une décision quant à l'opportunité de tenir une deuxième Conférence des ministres de l'Économie et des Finances ou d'autres décideurs économiques de l'espace francophone" (Déclaration de Québec, paragraphe 37, http://www.francophonie.org/IMG/pdf/decl-quebec-20083.pdf [26 janvier 2010])...

Tristan Leperlier École Normale Supérieure

Paris IV Sorbonne Sciences Po Paris

\section{Lise Bizzoni et Cécile Prévost-Thomas (dir.), avec la collaboration de Dany Saint-Laurent La chanson francophone engagée Montréal, Triptyque, 2008.}

Les premières années du nouveau millénaire auront été plutôt fécondes en monographies consacrées à des chanteurs ou chanteuses, de même qu'en publications savantes sur la chanson d'expression française. Que l'on songe à Plume Latraverse, masqué / démasqué de Mario Leduc (2003), à La chanson réaliste de Catherine Dutheil (2004), aux Mots de Barbara (2004) et à l'Esthétique de la chanson française contemporaine de Joël July (2007) ou à 